\title{
Compliance Adjustment of a Gripper System Designed for the Seizing of Objects of Modifiable Consistency
}

\author{
Andrea DEACONESCU \\ Transilvania University of Braşov, Romania, deacon@unitbv.ro
}

\begin{abstract}
The necessity of seizing objects of unknown forms and/or consistency entails conceiving gripper systems whose design and control strategies are oriented towards the capacity of safe physical handling. The paper presents and discusses such a soft gripper system that includes a pneumatic muscle as its actuation motor. The compliance of the entire system is achieved by modifying air pressure in the pneumatic muscle within a closed loop, thus ensuring the safe gripping of deformable objects.
\end{abstract}

\section{Keywords}

pneumatic muscle, gripper system, compliance

\section{Introduction}

In robotics gripping entails the contact between the effector element of a robot (the gripper) and an object in view of its manipulation. Grippers are components of robotic systems that facilitate the temporary contact with the object to be manipulated ensuring its position and orientation during transport and assembly.

Robotic systems whose design and control strategies are oriented towards the capacity of achieving safe physical human-robot interactions (soft robots) are conceived by biomimetic principles and are made from soft and/or smart materials following the functional morphology of living organisms. The soft nature allows the robots to adapt to their surroundings, to perform different and even autonomous tasks, and to mimic the motion and function of biological systems, such as human muscles $[1,2]$.

The continuous development of soft robot applications is due to the need of solving problems beyond the capability of classical robots built from rigid bodies. The concept of soft robotics entails the use of soft, deformable and optionally elastic structures, capable of functioning in uncertain and dynamic task environments, as well as of gripping objects of unknown forms and consistencies [3, 4].

In the case of rigid grippers, a large force applied to the object can deform or even destroy it (Figure 1). Without any tactile feedback or object recognition, the human operator needs to control and limit the force, so that gripping does not damage the object.
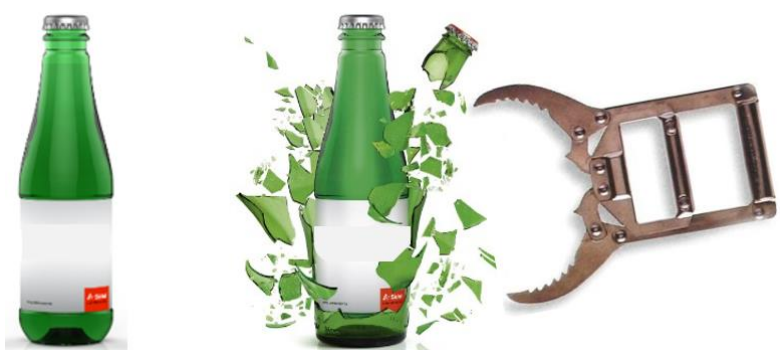

Fig. 1. Applying a not controlled force to gripping an object

Unlike rigid systems, the components of soft grippers display an adaptive behavior due to the low rigidity materials they are made from, what allows an inherently safe and adaptive contact with both solid and soft surfaces.

Avoiding unwanted situations like the one in Fig. 1 is possible by using for example soft fluidic actuation that ensures the adaptability of the gripper to a concrete work situation. In this case the 
retention forces of a deformable object are developed by controlling the pressure of a fluid fed to a closed chamber. The form of the pressured chamber causes its deformation by a preferential direction, the generated displacement being either lengthening or contraction [5].

For robotic seizing and gripping of fragile or deformable objects an alternative to highly sensorized grippers and complex control schemes is using adjustable compliant actuators (ACAs). In such a system the presence of sensors or complex controllers is not necessary, as it is the mechanical system itself that provides the required adaptive behavior [6]. The adaptive behavior of a gripper means the capability of damage-free handling of objects of unknown form and consistency or the capability to adjust to constraints generated by its work environment.

The paper presents and discusses a constructive solution of a gripper system that is capable of varying its behavior continuously between two limits, from soft to rigid and vice-versa. The proposed system uses a pneumatic muscle as actuation motor and a rack and pinion mechanical transmission in order to generate the required work motions. Further discussed is the effect of modifying the work pressure, namely the continuous adjustment of the system's compliance that enables the gripper to manipulate objects of different consistencies.

\section{Construction of the Gripper System}

The proposed system is of parallel asymmetrical type with two mobile jaws as illustrated in Figure 2 [7].

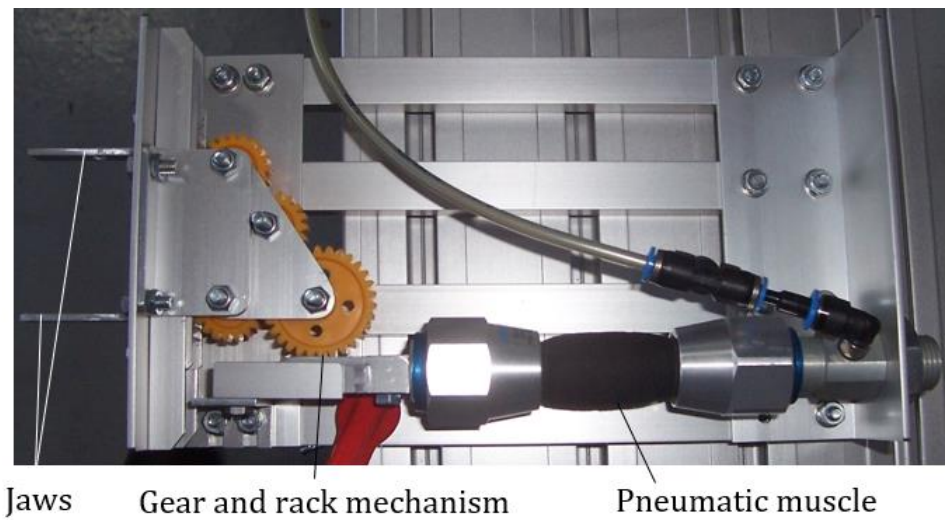

Fig. 2. Construction of the gripper system

The motor element of the gripper is a pneumatic muscle of type MAS-10-45N-AA-MC-O-ER-EG, manufactured by Festo. Upon being fed compressed air the muscle displaces a rack that drives a train of gears and racks on which the supporting arms of the jaws are fixed rigidly. The contact forces developed between the jaws and the seized object depend on the actuation force of the pneumatic muscle and on the dimensional characteristics of the mechanical system.

The utilized pneumatic muscle has an interior diameter of $10 \mathrm{~mm}$, a $45 \mathrm{~mm}$ long active part and is capable of generating a maximum force of $520 \mathrm{~N}$ when charged with air at a pressure of 6 bar. Taking into consideration the transmission function of the forces determined in [8], the maximum available force at each of the gripper's jaws is of $F_{\text {jaw_max }}=83.36 \mathrm{~N}$. Under these circumstances the gripper is capable of seizing and maintaining objects of a maximum mass of:

$$
m=\frac{\mu \cdot 2 \cdot F_{\text {jaw_max }}}{\left(g+a_{s}\right) \cdot S}=\frac{0.2 \cdot 2 \cdot 83.36}{(9.81+10) \cdot 2.5}=0.673 \mathrm{~kg}
$$

The above equation includes the following notations: gravitational acceleration: $g=9.81 \mathrm{~m} / \mathrm{s}^{2}$; emergency stop acceleration (deceleration): $a_{S}=10 \mathrm{~m} / \mathrm{s}^{2}$; friction coefficient between the jaw and the object: $\mu=0.2$; safety coefficient: $S=2.5$.

Figure 3 shows the pneumatic control diagram of the gripper system. The pneumatic control diagram of the muscle includes a proportional pressure regulator that receives an electric signal from either a reference module, or an external potentiometer. This allows for continuous modification of the regulator's feed pressure, causing the permanent variation of air pressure in the pneumatic muscle. This 
is a pressure - based control scheme where the pressure within the pneumatic muscle is measured and controlled in a closed loop. The possibility of continuously modifying the air pressure ensures the variation of the entire system's compliance.

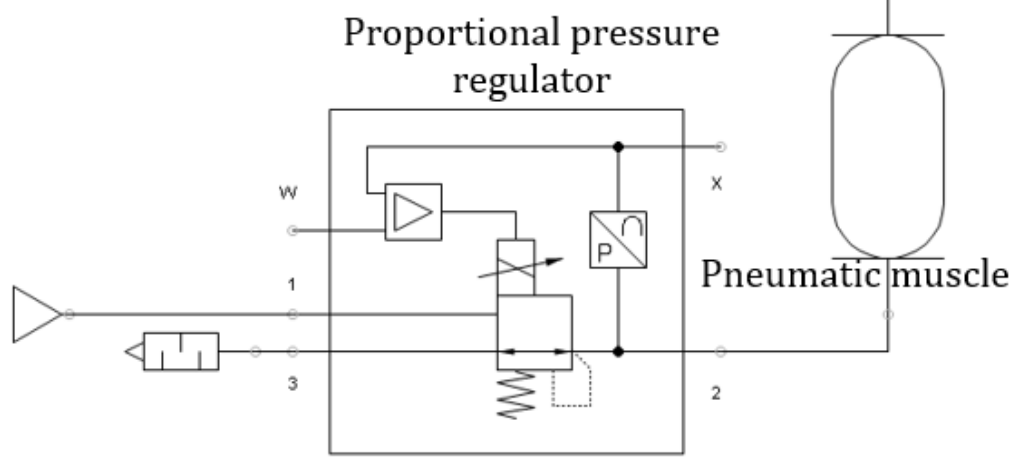

Fig. 3. Control of the soft gripper system

\section{Experimental Research}

The conducted experimental studies were aimed mainly at determining a method for the adjustment of the gripper system's compliance by the adaptive modification of the compressed air pressure. It is known that in order to be compliant a system requires a non-linear dependency of the developed force in relation to the conducted stroke. In order to verify this a measurement diagram was devised of the force developed by a jaw when the pneumatic muscle is fed air at various pressures (Figure 4) [9].

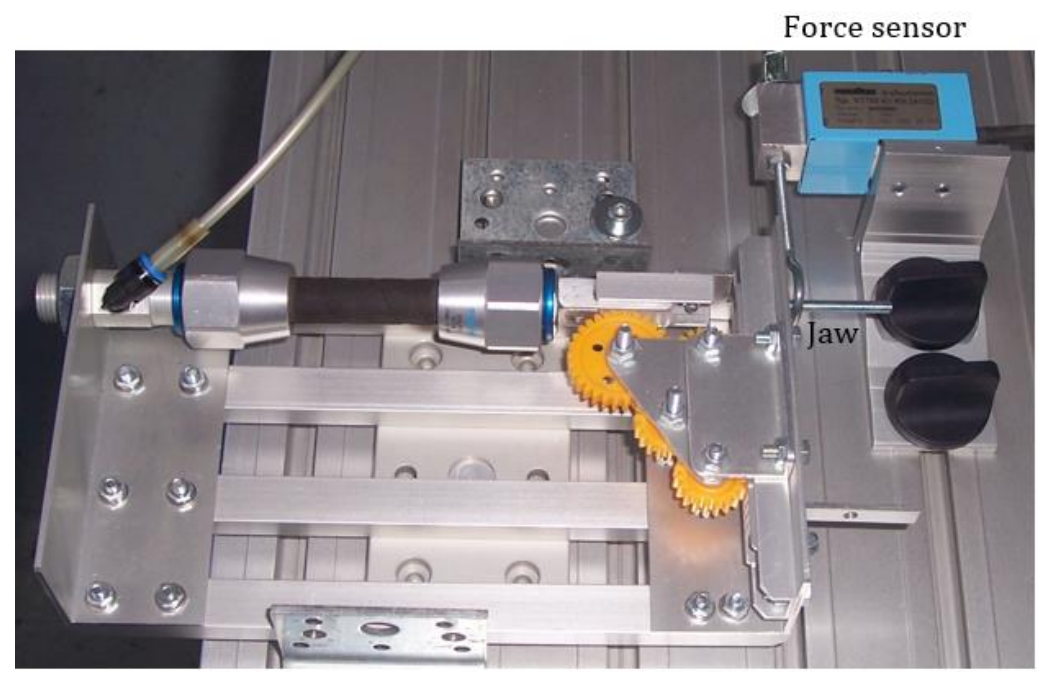

Fig. 4. Force measurement developed by one jaw

Table 1 shows the collected measured data and Figure 5 shows the graph of the variation of force $F_{\text {jaw }}$ versus $s$ - the stroke carried out by the rack (the axial deformation of the pneumatic muscle).

Table 1. Dependence of the force developed by one jaw on the stroke of the rack

(the axial deformation of the pneumatic muscle)

\begin{tabular}{|c|c|c|c|c|c|c|c|}
\hline \multirow{2}{*}{$\begin{array}{c}\mathrm{s} \\
{[\mathrm{mm}]}\end{array}$} & $F_{\text {jaw_6bar }}$ & $\mathrm{F}_{\text {jaw_4bar }}$ & $\mathrm{F}_{\text {jaw_zbar }}$ & \multirow{2}{*}{$\begin{array}{c}\mathrm{s} \\
{[\mathrm{mm}]}\end{array}$} & \multirow{2}{*}{\multicolumn{3}{|c|}{$\begin{array}{c}\mathrm{F}_{\text {jaw_4bar }} \\
{[\mathrm{N}]}\end{array}$}} \\
\hline & \multicolumn{3}{|c|}{$[\mathrm{N}]$} & & & & \\
\hline 0 & 83.36 & 58.12 & 29.97 & 4 & 33.02 & 15.6 & - \\
\hline 1 & 62.34 & 39.16 & 13.27 & 5 & 26.31 & 10.73 & - \\
\hline 2 & 49.95 & 28.71 & 5.15 & 6 & 20.29 & - & - \\
\hline 3 & 40.71 & 21.39 & - & & & & \\
\hline
\end{tabular}




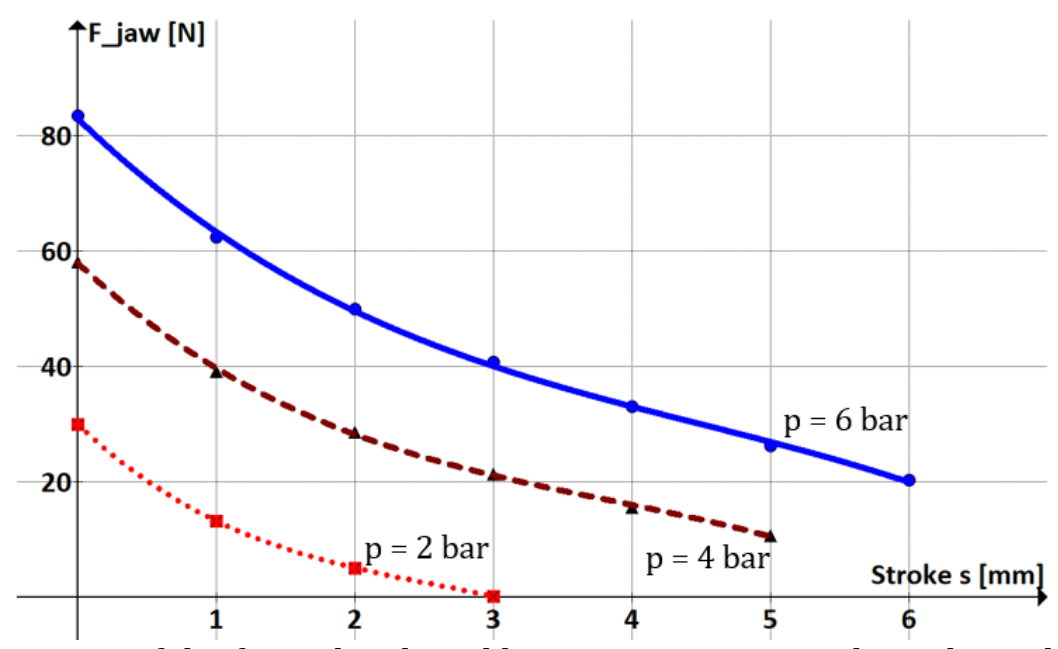

Fig. 5. Variation of the force developed by one jaw versus rack stroke and pressure

The functions that describe most accurately the evolution of the measured forces (correlation coefficient $\mathrm{R}^{2}>0.999$ ) are of the form of Eq. (2):

$$
F_{j a w}(s)=a_{1} \cdot s^{3}+a_{2} \cdot s^{2}+a_{3} \cdot s+a_{4},
$$

where the values of the coefficients $a_{1 \ldots}$ are given in Table 2 .

Table 2. Values of the coefficients of the polynomial regression function

\begin{tabular}{|c|c|c|c|c|}
\hline & $a_{1}$ & $a_{2}$ & $a_{3}$ & $a_{4}$ \\
\hline $\mathrm{p}=6$ bar & -0.2808 & 3.7954 & -23.1595 & 82.9714 \\
\hline $\mathrm{p}=4$ bar & -0.3958 & 4.5596 & -22.376 & 57.9386 \\
\hline $\mathrm{p}=2$ bar & -0.9033 & 7 & -22.7967 & 29.97 \\
\hline
\end{tabular}

Figure 5 shows a descending non-linear evolution of the developed force as the stroke carried out by the jaw increases. Consequently, a variable rigidity $k$ and implicitly a variable compliance $C$ are obtained, described by Eq. (3) and Eq. (4):

$$
\begin{gathered}
k=-\frac{d F_{j a w}}{d s}=-\left(3 \cdot a_{1} \cdot s^{2}+2 \cdot a_{2} \cdot s+a_{3}\right) \\
C=k^{-1}=\frac{1}{-\left(3 \cdot a_{1} \cdot s^{2}+2 \cdot a_{2} \cdot s+a_{3}\right)}
\end{gathered}
$$

Figures 6 and 7 show the variations of rigidity and compliance versus the stroke carried out by the jaw (the axial deformation of the pneumatic muscle) and the feed pressure.

The two graphs show that rigidity has a decreasing trend while compliance displays an increasing one as the gripper jaws approach the targeted object. This tendency is manifest only during the first interval of the strokes, thus limiting the operational range of the gripper. As a consequence of this behavior the deployment of the gripper is restricted to strokes up to $2.5 \mathrm{~mm}$ for small working pressures ( 2 bar) and to strokes up to $4.5 \mathrm{~mm}$ for a pressure of 6 bar.

It is important to point out that as the moment of contact and firm retention of the object is approached, due to the approximately concave shape of the compliance curve its gradient becomes increasingly abrupt and consequently the compliance of the gripper reaches high values within a short time.

The graph of Figure 7 also shows that once contact has been achieved with the object, different values of the compliance can be ensured by adequately adjusting the work pressure. The example illustrated in the figure shows that for an imposed jaw stroke of $2 \mathrm{~mm}$, by lowering air pressure from 6 to 2 bar the compliance increases about 2 times, from 0.0881 to $0.1774 \mathrm{~mm} / \mathrm{N}$. The higher values of the compliance are, the more adequate the gripper is for seizing delicate, fragile, easily deformable objects. 


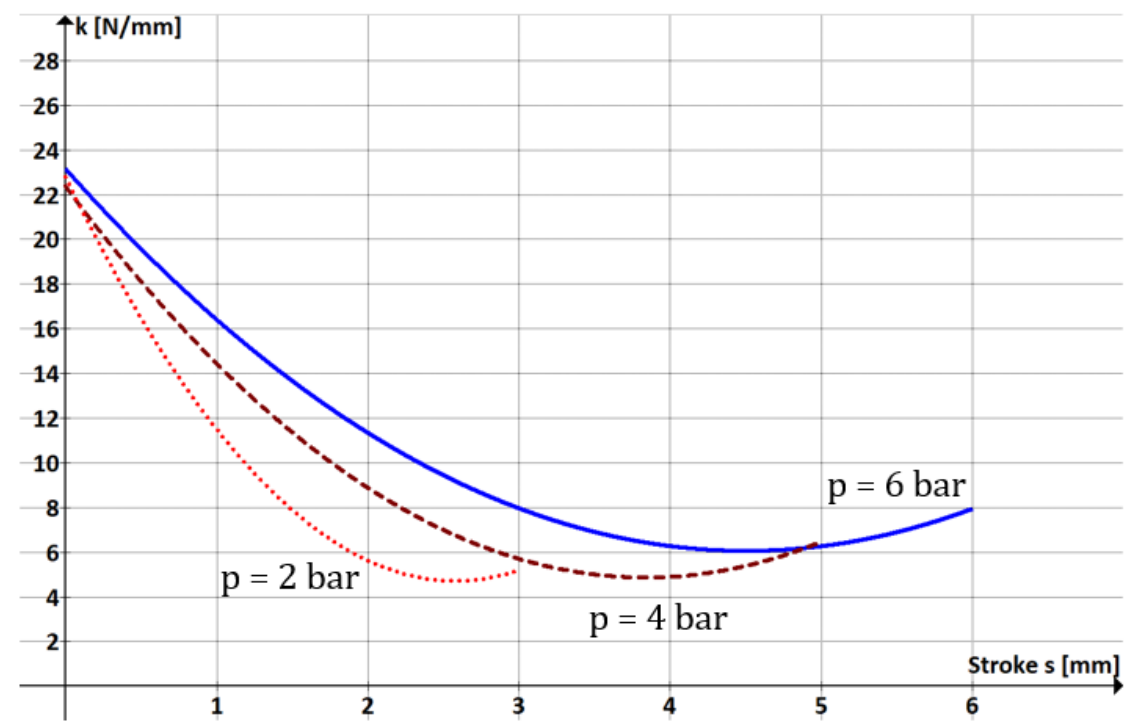

Fig. 6. Variation of rigidity versus jaw stroke and air pressure

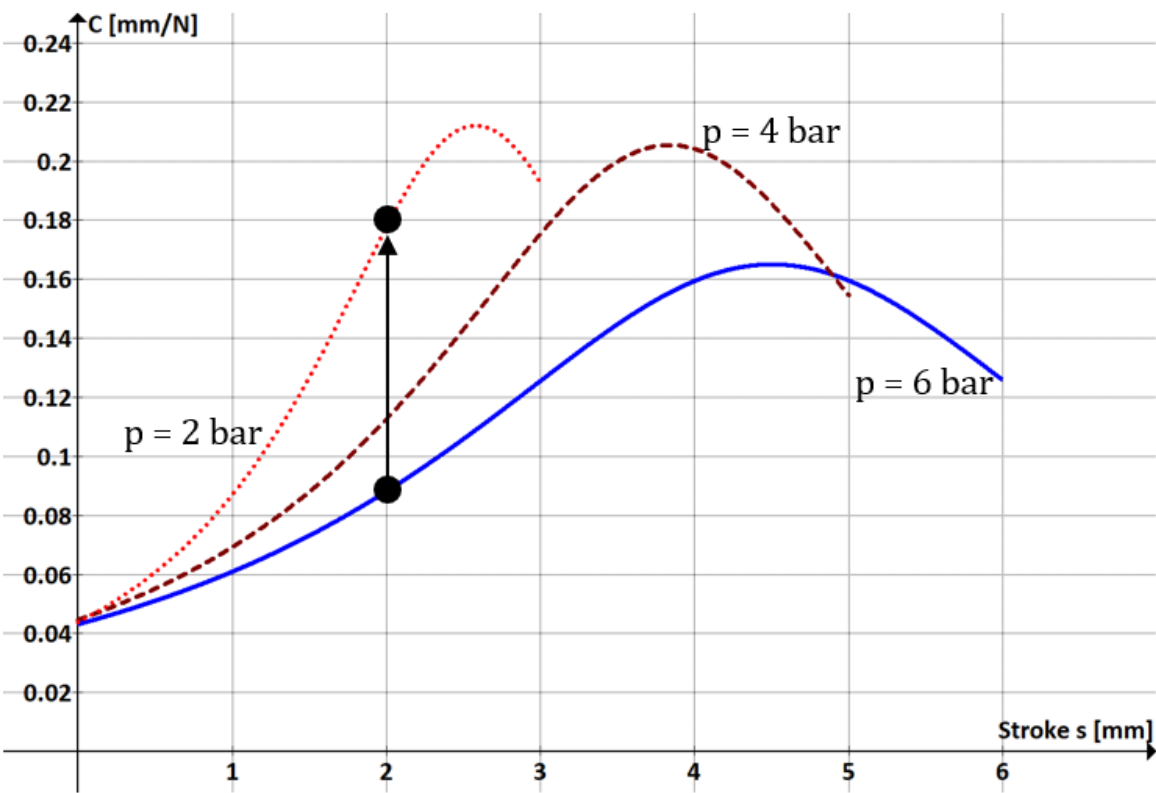

Fig. 7. Variation of compliance versus jaw stroke and air pressure

\section{Case Study}

The gripper has to retain a $250 \mathrm{ml}$ juice bottle filled with liquid (Figure 8) of a total mass of $M=0.269 \mathrm{~kg}, D_{e}=52 \mathrm{~mm}$ exterior diameter and $g=0.3 \mathrm{~mm}$ wall thickness. The bottle is made from polyethylene terephthalate (PET) with an elasticity module $E=5 \mathrm{GPa}$. The necessary force for retaining the bottle is computed by Eq. (1): $F_{\text {jaw }}=33.3 \mathrm{~N}$.

The accepted value of the radial deformation of the bottle wall at the time of its being seized by the gripper is imposed at $\Delta g=1 \mathrm{~mm}$. At this magnitude of the deformation while being gripped firmly the bottle is not destroyed. The deformation of the bottle is compensated by an additional displacement of each jaw by $1 \mathrm{~mm}$, from $s=0.5 \mathrm{~mm}$ to $s=1.5 \mathrm{~mm}$. Maintaining the gripping force constant $\left(F_{\text {jaw }}=33.3 \mathrm{~N}\right)$ requires the feed pressure of the pneumatic muscle to increase from 2.97 bar to 3.99 bar (Figure 9).

Figure 10 shows the variation of the system's compliance for this modification of the pressure. An increase of compliance with the deformation of the seized object can be noticed (from 0.0568 to 0.0979 $\mathrm{mm} / \mathrm{N}$ ), even at a high level of the pressure. 


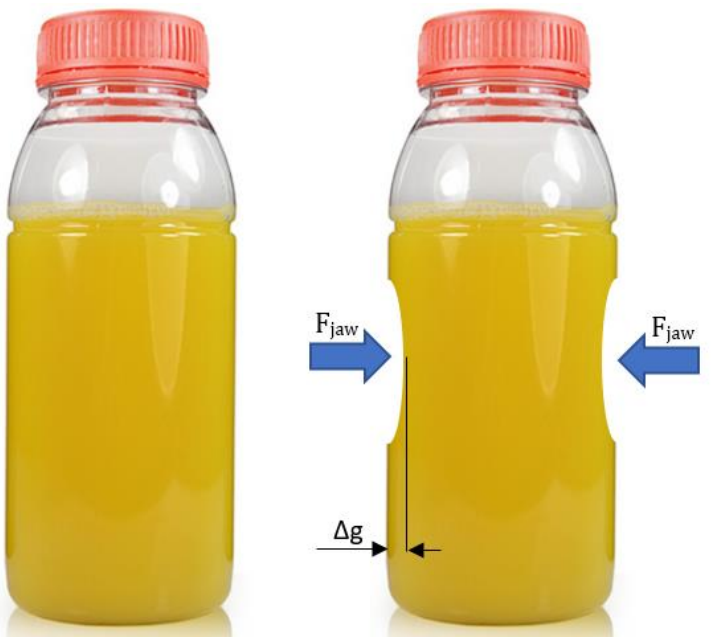

Fig. 8. Deformation of the juice bottle

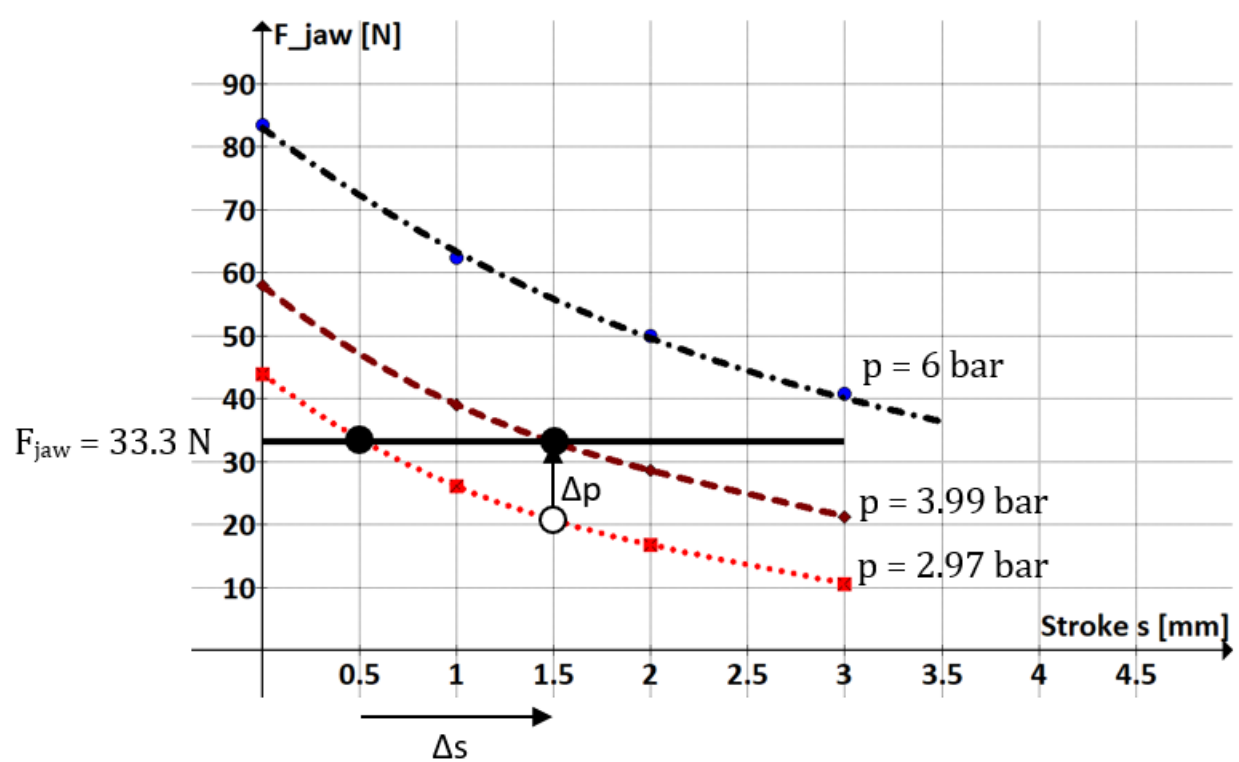

Fig. 9. Modifying the pressure to compensate the displacement of the jaw

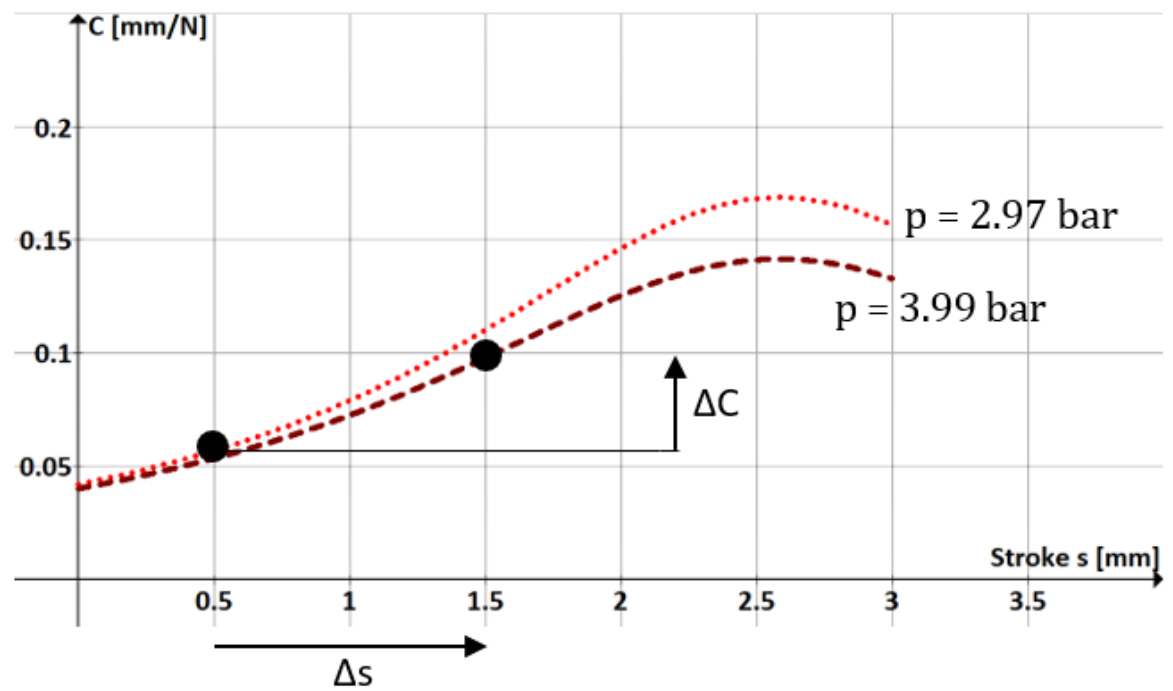

Fig. 10. Increase of compliance with the compensation of the jaw stroke 
The obtained results confirm that the gripper system proposed in this paper benefits from adjustable compliance that can be adapted to the concrete work situation.

\section{Conclusion}

Ensuring the adaptability of a gripper to the form and/or consistency of the object to be seized requires the utilization of soft gripper systems driven by adjustable compliant actuators (ACAs). The pneumatic muscle is such an actuator that was used in the concept of a gripper system with two parallel mobile jaws capable of seizing easily deformable objects without destroying them. The paper presents the characteristics of the gripper system as to the achievable forces, rigidity and compliance. The compliance of the system is adjusted by modifying the pressure of the air that is fed to the pneumatic muscle, monitored and controlled in a closed loop, by means of a proportional pressure regulator.

\section{References}

1. Schmitt F., Piccin O., Barbé L., Bayle B. (2018): Soft Robots Manufacturing: A Review. Frontiers in Robotics and AI, e-ISSN 2296-9144, https://doi.org/10.3389/frobt.2018.00084

2. *** (2018): The soft touch of robots. Nature Reviews Materials, ISSN 2058-8437, e-ISSN 2058-8437, Vol. 3, is. 71, https://doi.org/10.1038/s41578-018-0017-8

3. Marshall B. (2018): Soft Robot Design with Biomimetics. Available at: https://www.rsonline.com/designspark/soft-robot-design-with-biomimetics Accessed: 2020-04-12

4. Iida F., Laschi C. (2011): Soft Robotics: Challenges and Perspectives. Procedia Computer Science, ISSN 1877-0509, Vol. 7, p. 99-102, https://doi.org/10.1016/i.procs.2011.12.030

5. Digumarti K.M., Conn A.T., Rossiter J. (2017): Euglenoid-inspired giant shape change for highly deformable soft robots. IEEE Robotics and Automation Letters, e-ISSN 2377-3766, Vol. 2, is. 4, p. 2302-2307 doi: 10.1109/LRA.2017.2726113

6. Deaconescu T., Deaconescu A. (2017): Pneumatic Muscle Actuated Adjustable Compliant Gripper System for Assembly Operations. Strojniški vestnik - Journal of Mechanical Engineering, e-ISSN 2536-2948, Vol. 63, no. 4, p. 225-234, DOI: $10.5545 /$ sv-jme.2016.4239

7. Deaconescu T., Deaconescu A., Negrea D. (2019): Sistem de prehensiune paralel cu două bacuri, asimetric, acţionat cu muşchi pneumatici (Asymmetrical Parallel Gripper System with Two Jaws Actuated by Pneumatic Muscles). Patent R0 130331 B1, Assigned to Transilvania University of Braşov (in Romanian)

8. Negrea D., Deaconescu A., Deaconescu T. (2014): Actuation by Pneumatic Muscles of a Parallel Asymmetric Gripper System. Applied Mechanics and Materials, ISSN 1662-7482, Vol. 548-549, p. 943-947, https://doi.org/10.4028/www.scientific.net/AMM.548-549.943

9. Sârbu F., Deaconescu A., Deaconescu T. (2019): Adjustable compliance soft gripper system. International Journal of Advanced Robotic Systems, ISSN 1729-8814, eISSN 1729-8814, July-August 2019, p. 1-10, https://doi.org/10.1177/1729881419866580 